\title{
Stability and Stabilization of Discrete-Time Semi-Markov Jump Linear Systems via Semi-Markov Kernel Approach
}

\author{
Lixian Zhang, Yusong Leng, and Patrizio Colaneri, Fellow, IEEE
}

\begin{abstract}
This technical note is concerned with the problems of stability and stabilization for a class of discrete-time semiMarkov jump linear systems (S-MJLSs). The discrete-time semiMarkov kernel (SMK) is introduced, where the probability density function of sojourn-time is dependent on both current and next system mode. As a consequence, different types of distributions and/or different parameters in a same type of distribution of sojourn-time, depending on the target mode towards which the system jumps, can coexist in each mode of a SMK. The underlying S-MJLSs are therefore more general than those considered in existing studies. A new stability concept generalizing the traditional mean-square stability is proposed such that numerically testable criteria on the basis of SMK are obtained. Numerical examples are presented to illustrate the validity and advantage of the developed theoretical results.
\end{abstract}

Index Terms-Mean-square stability, semi-Markov jump linear systems, semi-Markov kernel, sojourn-time.

\section{INTRODUCTION}

The past decades have seen a great advance in theories and applications of Markov jump linear systems (MJLSs). The systems can effectively model dynamical processes involved with stochastic switching (generally autonomous) subject to a Markov chain. Typical examples include fault-tolerant control systems where abrupt faults occur randomly and networked control systems where networkinduced communication imperfections vary in a stochastic way, see for example [1] and [2]. The primary concern of MJLSs is the stability analysis that is relatively challenging due to the hybrid nature of the system. Up to date, quite a few important stability notions, stochastic stability, mean-square stability (MSS), almost sure stability and so on, are persistently utilized and many results are rather fundamental in the field of stochastic switching systems, see for example, [3] and [4]. Other issues including control, estimation, model reduction of MJLSs and the underlying systems with various complex dynamics, timedelays, uncertainties, positiveness, etc., have also been intensively studied, see for example, [5]-[7] and the references therein.

However, as pointed out in [8]-[12], although Markov processes or Markov chain do have the ability in describing the mode switching in

Manuscript received April 24, 2014; revised March 4, 2015; accepted May 20, 2015. Date of publication June 1, 2015; date of current version January 26, 2016. This work was supported in part by the National Natural Science Foundation of China under Grant 61322301, the National Natural Science Foundation of Heilongjiang under Grants F201417 and JC2015015, and the Fundamental Research Funds for the Central Universities, China HIT.BRETIII.201211 and HIT.BRETIV.201306. Recommended by Associate Editor C. Belta.

L. Zhang and Y. Leng are with the School of Astronautics, Harbin Institute of Technology, Harbin 150080, China (e-mail: lixianzhang @ hit.edu.cn; yusongleng@hit.edu.cn).

P. Colaneri is with the Dipartimento di Elettronica, Informazione e Bioingegneria, Politecnico di Milano and CNR-IEIIT, 20133 Milano, Italy (e-mail: colaneri@elet.polimi.it).

Color versions of one or more of the figures in this paper are available online. many practical applications, they cannot cover all the scenarios yet. A key restriction in the underlying MJLSs is that the sojourn-time (the interval between two consecutive jumps) of each subsystem is subject to exponential distribution (geometric distribution in discretetime domain, respectively). To relax the restriction, the concepts of nonhomogeneous Markov chain and semi-Markov chain, where the transition probabilities (TPs) are time-varying and memory, respectively, have been introduced in the control community, and studies on the corresponding systems have been gradually launched. As for the former case that is relatively tractable, the systems with dwell-time ${ }^{1}$ switching TPs [10], piecewise homogeneous TPs [11], and polytopic time-varying TPs [12], etc., have been proposed within the past few years, and various methodologies have been explored for different situations. Such proposals have provided a basic foundation for further studies on the systems with nonhomogeneous TPs.

On the other hand, the developed theories on semi-Markov jump linear systems (S-MJLSs) are far away from maturity yet, although the systems have been investigated since 1960s, see for example, [8], [9], [13] and [14]. The inherent difficulties mainly lie in how the probability density function (PDF) information of the sojourntime can be completely used in deriving criteria of stability analysis and control synthesis, and further, how the obtained criteria can be numerically tested. With the assumption that the PDF is dependent on the current system mode, the stabilization problem for a class of continuous-time S-MJLSs has been addressed in [8] by solving a set of coupled algebraic Riccati equations. Further improvements achieved in [9] offer a framework under which the control problems can be solved by the techniques of linear matrix inequalities (more conveniently to be checked), with a priori information of the upper and lower bounds of a PDF. However, it is noted that almost all the existing results presume that each mode possesses a single distribution of sojourn-time with certain parameters in a semi-Markov chain. It is very likely that the types of distributions and/or the parameters in a same type of distribution of sojourn-time can be different for each mode, depending on the target mode to which the system jumps from the current mode.

Motivated by the above observations, in this technical note, we aim at addressing the problems of stability and stabilization for a class of discrete-time S-MJLSs. There are two main contributions. First, the concept of discrete-time semi-Markov kernel (SMK) is introduced, and the PDF of sojourn-time in the SMK is allowed to depend on both the current and next system mode. As a result, different parameters in a same type of distribution or even different types of distributions of sojourn-time can be simultaneously considered in each mode of a SMK depending on the target mode, and the underlying S-MJLSs are therefore more general than those studied previously. Second, a new stability concept called $\sigma$-error mean square stability ( $\sigma$-MSS) is proposed, where $\sigma$ is used to characterize the degree of "approximation error" of $\sigma$-MSS to MSS (without the error the underlying system holds MSS). The stability concept generalizes the traditional MSS,

\footnotetext{
${ }^{1}$ A term commonly used in the context of nondeterministic switched systems, where dwell-time is generally no greater than the running time (or sojourn-time called in this technical note) of an activated subsystem.
} 


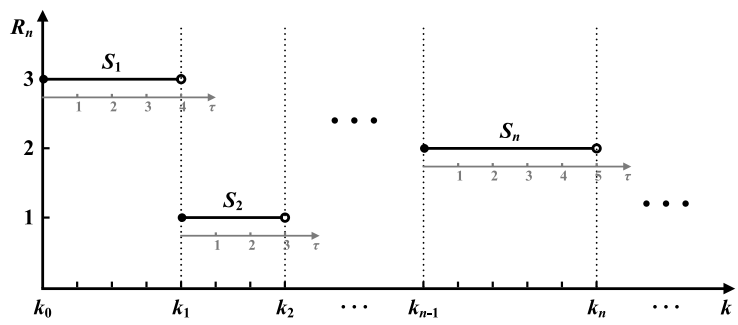

Fig. 1. Illustration of stochastic processes $R_{n}, k_{n}$ and $S_{n}(M=3)$.

based on which the numerically testable stability and stabilization criteria that explicitly contains the PDF information of sojourn-time can be obtained.

Notations: In this technical note, $\mathbb{R}^{n}$ denotes the $n$-dimensional Euclidean space; $\|\cdot\|$ refers to the Euclidean vector norm; $\mathbb{R}_{+}$and $\mathbb{Z}_{+}$ denote the set of non-negative real numbers and set of non-negative integers, respectively; $\mathbb{R}_{\left[s_{1}, s_{2}\right]}, \mathbb{Z}_{\geq s_{1}}$ and $\mathbb{Z}_{\left[s_{1}, s_{2}\right]}$ denote the sets $\{k \in$ $\left.\mathbb{R}_{+} \mid s_{1} \leq k \leq s_{2}\right\},\left\{k \in \mathbb{Z}_{+} \mid k \geq s_{1}\right\}$ and $\left\{k \in \mathbb{Z}_{+} \mid s_{1} \leq k \leq s_{2}\right\}$, respectively. For notation $(\Psi, \mathcal{F}, \operatorname{Pr}), \Psi$ represents the sample space, $\mathcal{F}$ is the $\sigma$-algebra of subsets of the sample space and $\operatorname{Pr}$ is the probability measure on $\mathcal{F}$. $\mathcal{C}^{1}$ denotes the space of continuously differentiable functions, and a function $\kappa:[0, \infty) \rightarrow[0, \infty)$ is said to be of class $\mathcal{K}_{\infty}$ if it is continuous, strictly increasing, unbounded, and $\kappa(0)=0$. In addition, $\operatorname{diag}\{\cdots\}$ stands for a block-diagonal matrix and $\operatorname{diag}_{(n)}\{X\}$ a $n \times n$ block-diagonal matrix where all diagonal entries are $X$. Symbol $*$ is used as an ellipsis for the terms that are introduced by symmetry.

\section{Preliminaries And Problem Formulation}

Fix the complete probability space $(\Psi, \mathcal{F}, \operatorname{Pr})$ and consider the following discrete-time stochastic switching systems:

$$
x(k+1)=A\left(r_{k}\right) x(k)+B\left(r_{k}\right) u(k)
$$

where $x(k) \in \mathbb{R}^{n}, u(k) \in \mathbb{R}^{n_{u}}$ are the system state and control input, respectively. $\left\{r_{k}\right\}_{k \in \mathbb{Z}_{+}}$is a stochastic process, considered to be a semi-Markov chain, which takes values in a finite set $\mathcal{I} \triangleq$ $\{1,2, \ldots, M\}$, and governs the switching among $M$ system modes. For $r_{k}=i \in \mathcal{I}$, the pair of matrices of the $i$ th system mode is denoted by $\left(A_{i}, B_{i}\right)$, which are real known matrices with appropriate dimensions.

To introduce the semi-Markov chain formally, we shall recall two concepts on Markov renewal chain and semi-Markov kernel, for which three following stochastic processes are first needed:

i) The stochastic process $\left\{R_{n}\right\}_{n \in \mathbb{Z}_{+}}$taking values in $\mathcal{I}$, where $R_{n}$ is the index of system mode at the $n$th jump;

ii) The stochastic process $\left\{k_{n}\right\}_{n \in \mathbb{Z}_{+}}$taking values in $\mathbb{Z}_{+}$, where $k_{n}$ denotes the time at the $n$th jump. It is noted that $k_{0}=0$ and $k_{n}$ increases monotonically with $n$;

iii) The stochastic process $\left\{S_{n}\right\}_{n \in \mathbb{Z}_{+}}$taking values in $\mathbb{Z}_{+}$, where $S_{n}=k_{n}-k_{n-1}, \forall n \in \mathbb{Z}_{\geq 1}$ denotes the sojourn-time of mode $R_{n-1}$ between the $(n-1)$ th jump and $n$th jump, and $S_{0}=0$.

These stochastic processes are illustrated in Fig. 1, and more details can be found in [15] and the references therein.

Definition 1 [15]: The stochastic process $\left\{\left(R_{n}, k_{n}\right)\right\}_{n \in \mathbb{Z}_{+}}$is said to be a discrete-time homogeneous Markov renewal chain (MRC) if for any $j \in \mathcal{I}, \tau \in \mathbb{Z}_{+}$and $n \in \mathbb{Z}_{+}, \operatorname{Pr}\left(R_{n+1}=j, S_{n+1}=\right.$ $\left.\tau \mid R_{0}, \ldots, R_{n}=i ; k_{0}, \ldots, k_{n}\right)=\operatorname{Pr}\left(R_{n+1}=j, S_{n+1}=\tau \mid R_{n}=i\right)$ $=\operatorname{Pr}\left(R_{1}=j, S_{1}=\tau \mid R_{0}=i\right)$.

Then, let $\pi_{i j}(\tau) \triangleq \operatorname{Pr}\left(R_{n+1}=j, S_{n+1}=\tau \mid R_{n}=i\right), \forall i, j \in \mathcal{I}$, $\forall \tau \in \mathbb{Z}_{+}$, the matrix $\Pi(\tau)=\left[\pi_{i j}(\tau)\right]_{i, j \in \mathcal{I}}$ is called discrete-time semi-
Markov kernel (SMK), where $\pi_{i j}(\tau) \in \mathbb{R}_{[0,1]}$ and $\sum_{\tau=0}^{\infty} \sum_{j \in \mathcal{I}}$ $\pi_{i j}(\tau)=1$ with $\pi_{i j}(0)=0$. In addition, from [15], $\left\{R_{n}\right\}_{n \in \mathbb{Z}_{+}}$is called the embedded Markov chain (EMC) of MRC $\left\{\left(R_{n}, k_{n}\right)\right\}_{n \in \mathbb{Z}_{+}}$, and the TPs matrix $\Theta=\left[\theta_{i j}\right]_{i, j \in \mathcal{I}}$ of $\left\{R_{n}\right\}_{n \in \mathbb{Z}_{+}}$is defined by $\theta_{i j} \triangleq \operatorname{Pr}\left(R_{n+1}=j \mid R_{n}=i\right), \forall i, j \in \mathcal{I}$ with $\theta_{i i}=0$.

With the above concepts, the definition of semi-Markov chain is given as below.

Definition 2 [15]: Consider a MRC $\left\{\left(R_{n}, k_{n}\right)\right\}_{n \in \mathbb{Z}_{+}}$. The chain $\left\{r_{k}\right\}_{k \in \mathbb{Z}_{+}}$is said to be a semi-Markov chain (SMC) associated with $\operatorname{MRC}\left\{\left(R_{n}, k_{n}\right)\right\}_{n \in \mathbb{Z}_{+}}$, if $r_{k}=R_{N(k)}, \forall k \in \mathbb{Z}_{+}$, where $N(k) \triangleq$ $\max \left\{n \in \mathbb{Z}_{+} \mid k_{n} \leq k\right\}$.

It is worth noting that the difference between EMC and SMC lies in that the stochastic variable varies with jump instant $k_{n}$ in the former, whereas with the sampling instant $k$ in the latter. From Definition 2, it is straightforward that the evolution of SMC is generated by the SMK $\Pi(\tau)$ that is dependent on sojourn-time $\tau$. Thus, the knowledge on the probability density function (PDF) of sojourn-time is required to characterize a SMC. In this technical note, the PDF depending on both the current and next system mode is considered and defined as $\omega_{i j}(\tau) \triangleq \operatorname{Pr}\left(S_{n+1}=\tau \mid R_{n+1}=j, R_{n}=i\right), \forall i, j \in \mathcal{I}, \forall \tau \in \mathbb{Z}_{+}$. As a consequence

$$
\begin{aligned}
\pi_{i j}(\tau) & =\frac{\operatorname{Pr}\left(R_{n+1}=j, R_{n}=i\right)}{\operatorname{Pr}\left(R_{n}=i\right)} \frac{\operatorname{Pr}\left(R_{n+1}=j, S_{n+1}=\tau, R_{n}=i\right)}{\operatorname{Pr}\left(R_{n+1}=j, R_{n}=i\right)} \\
& =\theta_{i j} \omega_{i j}(\tau) .
\end{aligned}
$$

Remark 1: Letting the PDF that only depends on the current system mode be denoted as $f_{i}(\tau)=\operatorname{Pr}\left(S_{n+1}=\tau \mid R_{n}=i\right), \forall i \in \mathcal{I}, \forall \tau \in$ $\mathbb{Z}_{+}$, it yields that $f_{i}(\tau)=\sum_{j \in \mathcal{I}} \pi_{i j}(\tau)=\sum_{j \in \mathcal{I}} \theta_{i j} \omega_{i j}(\tau)$. It can be observed that different $\omega_{i j}(\tau)$ may lead to a same $f_{i}(\tau)$. In almost all the previous studies on S-MJLSs, $f_{i}(\tau)$ is used to obtain the sojourntime-dependent transition probability at mode $i$, say, denoted as $\lambda_{i}(\tau)$, and further the TPs $\lambda_{i j}(\tau)$ by multiplying $\theta_{i j}$. Therefore the PDF $f_{i}(\tau)$ can be only one type, but $\omega_{i j}(\tau)$ that is used to form SMK in this technical note can be $r_{k+1}$ dependent, having different types of distributions or different parameters in a same type of distribution for any $i \in \mathcal{I}$. Therefore, the PDF considered in the technical note is more specific and capable of describing the corresponding SMC accurately rather than $f_{i}(\tau)$.

Throughout the technical note, the cumulative density function (CDF) of sojourn-time for the $i$ th system mode, $\forall i \in \mathcal{I}$, is denoted as $F_{i}(\tau)=\operatorname{Pr}\left(S_{n+1} \leq \tau \mid R_{n}=i\right)=\sum_{l=0}^{\tau} \sum_{j \in \mathcal{I}} \pi_{i j}(l)$, and it is assumed that $\omega_{i j}(0)=F_{i}(0)=0$ without loss of generality. Now, to present the purposes of this technical note more precisely, the following stability definitions are required.

Definition 3: Consider a discrete-time stochastic switching nonlinear system $x_{k+1}=f\left(x_{k}, r_{k}\right)$, where $r_{k}$ is a certain stochastic process governing the system switching and taking values in $\mathcal{I}$. The system is said to be mean-square stable if, for any initial conditions $x_{0} \in$ $\mathbb{R}^{n}, r_{0} \in \mathcal{I}$, the following holds:

$$
\left.\lim _{k \rightarrow \infty} \mathbb{E}\left[\|x(k)\|^{2}\right]\right|_{x_{0}, r_{0}}=0 .
$$

Remark 2: In Definition 3, when $r_{k}$ is a Markov chain and the underlying system is linear, we can typically find the corresponding version of Definition 3 in the literature of MJLSs, see for example, [10] and [16]. Nonetheless, one drawback of Definition 3 is that it is established allowing for the random sojourn-time to be any length (even infinity), regardless of the fact that the practical sojourn-time is generally finite. Therefore, letting $T_{\max }^{i}$ denote the upper bound of sojourn-time for the $i$ th mode of system (1), we generalize the MSS in this technical note to the following concept. 
Definition 4: System (1) with $u(k) \equiv 0$, is said to be $\sigma$-error meansquare stable if, for any initial conditions $x_{0} \in \mathbb{R}^{n}, r_{0} \in \mathcal{I}$ and the upper bound of sojourn-time $T_{\max }^{i} \in \mathbb{Z}_{\geq 1}, \forall i \in \mathcal{I}$, the following holds:

$$
\left.\lim _{k \rightarrow \infty} \mathbb{E}\left[\|x(k)\|^{2}\right]\right|_{x_{0}, r_{0}, S_{n+1} \leq\left. T_{\max }^{i}\right|_{R_{n}=i}}=0 .
$$

Further, $\sigma$ is defined as

$$
\sigma \triangleq \sum_{i \in \mathcal{I}}\left|\ln \left(F_{i}\left(T_{\max }^{i}\right)\right)\right| .
$$

Remark 3: Note that in Definition 4, $\sigma$ varies with $T_{\max }^{i}$ ( $\sigma$ decreases if all $T_{\max }^{i}$ are increased). In view of this, $\sigma$ is actually capable to characterize the degree of "approximation error" of $\sigma$-MSS to MSS. Particularly, when $T_{\max }^{i} \rightarrow \infty, \forall i \in \mathcal{I}$, which implies that $F_{i}\left(T_{\max }^{i}\right) \rightarrow 1$, then, $\sigma \rightarrow 0$ and accordingly the $\sigma$-MSS approximates to MSS without any error.

Then, the objectives in this technical note are to derive the $\sigma$-MSS criterion for system (1), and to design a state-feedback stabilizing controller guaranteeing the $\sigma$-MSS of the resulting closed-loop system. The mode-dependent controller is considered here with the form

$$
u(k)=K_{i} x(k), \quad \forall r_{k}=i \in \mathcal{I}
$$

where $K_{i}$ is the controller gain to be determined.

\section{MAin Results}

In this section, the numerically testable stability and stabilization criteria for S-MJLSs will be developed. A result on MSS of stochastic switching nonlinear systems is first given as below for later use.

Lemma 1: Consider a discrete-time stochastic switching nonlinear system $x_{k+1}=f\left(x_{k}, r_{k}\right)$, where $x_{k}$ and $r_{k}$ denote the system state and mode index, respectively. The switching instants are denoted by $k_{0}, k_{1}, \ldots, k_{s}, \ldots$ with $k_{0}=0$. The system is mean-square stable, if there exist a set of $\mathcal{C}^{1}$ functions $V\left(x_{k}, r_{k}\right): \mathbb{R}^{n} \rightarrow \mathbb{R}$ and three class $\mathcal{K}_{\infty}$ functions $\alpha_{1}, \alpha_{2}, \alpha_{3}$, such that for any initial conditions $x_{0} \in \mathbb{R}^{n}, r_{0} \in \mathcal{I}$ and a given finite $h_{i}>0, \forall r_{k_{s}}=i \in \mathcal{I}$

$$
\begin{aligned}
& \alpha_{1}\left(\left\|x_{k}\right\|\right) \leq V\left(x_{k}, r_{k_{s}}\right) \leq \alpha_{2}\left(\left\|x_{k}\right\|\right) \\
& V\left(x_{k}, r_{k_{s}}\right) \leq h_{i} V\left(x_{k_{s}}, r_{k_{s}}\right), \quad k \in \mathbb{Z}_{\left(k_{s}, k_{s+1}\right]} \\
& \left.\mathbb{E}\left[V\left(x_{k_{s+1}}, r_{k_{s+1}}\right)\right]\right|_{x_{0}, r_{0}}-V\left(x_{k_{s}}, r_{k_{s}}\right) \leq-\alpha_{3}\left(\left\|x_{k_{s}}\right\|\right) .
\end{aligned}
$$

Proof: It follows from the proof of [16, Theorem 1] that (9) ensures $\left.\sum_{s=0}^{\infty} \mathbb{E}\left[\alpha_{3}\left(\left\|x_{k_{s}}\right\|\right)\right]\right|_{x_{0}, r_{0}} \leq \mathbb{E}\left[V\left(x_{0}, r_{0}\right)\right]<\infty$ which implies $\left.\lim _{s \rightarrow \infty} \mathbb{E}\left[\alpha_{3}\left(\left\|x_{k_{s}}\right\|\right)\right]\right|_{x_{0}, r_{0}}=0$. Since $\quad \alpha_{3} \in \mathcal{K}_{\infty}, \quad \lim _{s \rightarrow \infty}$ $\left.\mathbb{E}\left[\left\|x_{k_{s}}\right\|^{2}\right]\right|_{x_{0}, r_{0}}=0$ holds. On the other hand, taking mathematical expectations at both sides of (7), (8), we have $\left.\mathbb{E}\left[\alpha_{1}\left(\left\|x_{k}\right\|\right)\right]\right|_{x_{0}, r_{0}} \leq$ $\left.h_{i} \mathbb{E}\left[V\left(x_{k_{s}}, r_{k_{s}}\right)\right]\right|_{x_{0}, r_{0}} \leq\left. h_{i} \mathbb{E}\left[\alpha_{2}\left(\left\|x_{k_{s}}\right\|\right)\right]\right|_{x_{0}, r_{0}}$. As $s \rightarrow \infty, k \rightarrow \infty$, therefore $\left.\lim _{k \rightarrow \infty} \mathbb{E}\left[\alpha_{1}\left(\left\|x_{k}\right\|\right)\right]\right|_{x_{0}, r_{0}} \leq\left. h_{i} \lim _{s \rightarrow \infty} \mathbb{E}\left[\alpha_{2}\left(\| x_{k_{s}} \mid\right)\right]\right|_{x_{0}, r_{0}}$, which implies (3).

Then, the following theorem gives a criterion of $\sigma$-MSS for unforced S-MJLSs.

Theorem 1: Consider S-MJLS (1) with $u(k) \equiv 0$ and a given finite constant $h_{i}>0$. If, $\forall i \in \mathcal{I}$, there exist $T_{\max }^{i} \in \mathbb{Z}_{\geq 1}$ and matrices $P_{i} \succ$ 0 such that $\forall t \in \mathbb{Z}_{\left[1, T_{\text {max }}^{i}\right]}$

$$
\begin{aligned}
A_{i}^{\prime t} P_{i} A_{i}^{t}-h_{i} P_{i} & \prec 0 \\
\sum_{\tau=1}^{T_{\max }^{i}} A_{i}^{\prime \tau} \mathcal{P}_{i}(\tau) A_{i}^{\tau}-P_{i} & \prec 0
\end{aligned}
$$

where $\mathcal{P}_{i}(\tau) \triangleq \sum_{j \in \mathcal{I}} \pi_{i j}(\tau) P_{j} / \eta_{i}$ with $\eta_{i} \triangleq \sum_{\tau=1}^{T_{\max }^{i}} \sum_{j \in \mathcal{I}} \pi_{i j}(\tau)$, then the system is $\sigma$-error mean-square stable.

Proof: Construct the Lyapunov function as $V_{i}\left(x_{k}\right) \triangleq$ $\left.V\left(x_{k}, R_{n}\right)\right|_{R_{n}=i}=x_{k}^{\prime} P_{i} x_{k}, \forall i \in \mathcal{I}$, where $P_{i}$ satisfies (10) and (11). First, it is straightforward that

$$
\inf _{i \in \mathcal{I}}\left\{\lambda_{\min }\left(P_{i}\right)\right\}\left\|x_{k}\right\|^{2} \leq V_{i}\left(x_{k}\right) \leq \sup _{i \in \mathcal{I}}\left\{\lambda_{\max }\left(P_{i}\right)\right\}\left\|x_{k}\right\|^{2}
$$

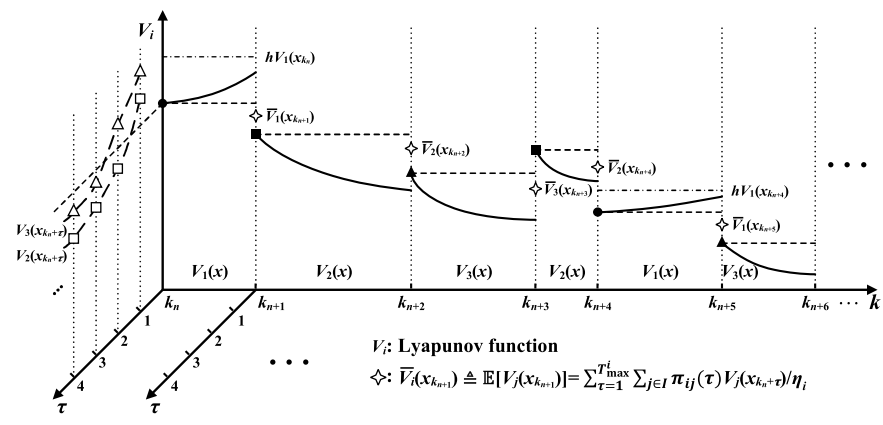

Fig. 2. Illustration of Theorem 1 for $M=3$. In $\left(k, V_{i}\right)$-coordinate, solid line shows the real evolution of Lyapunov function, and in $\left(\tau, V_{i}\right)$-coordinate, dashed line illustrates a possible evolution of the Lyapunov function starting from a fixed mode at a certain jumping instant; circles initiate $V_{1}$, squares initiate $V_{2}$ and triangles initiate $V_{3}$.

where $\lambda_{\min }\left(P_{i}\right)$ (respectively, $\lambda_{\max }\left(P_{i}\right)$ ) denotes the minimal (respectively, maximal) eigenvalue of $P_{i}$. In addition, for the case $R_{n}=$ $i$, the following is ensured by (10) $\forall t \in \mathbb{Z}_{\left[1, T_{\max }^{i}\right]}$

$$
V_{i}\left(x_{k_{n}+t}\right)-h_{i} V_{i}\left(x_{k_{n}}\right)=x_{k_{n}}^{\prime}\left(A_{i}^{\prime t} P_{i} A_{i}^{t}-h_{i} P_{i}\right) x_{k_{n}}<0 .
$$

On the other hand, for $R_{n}=i, R_{n+1}=j$, letting the sojourn-time $k_{n+1}-k_{n}$ be denoted by $\tau$, it follows from (11) that

$$
\begin{gathered}
\left.\mathbb{E}\left[V_{j}\left(x_{k_{n+1}}\right)\right]\right|_{x_{0}, r_{0}, S_{n+1} \leq\left. T_{\max }^{i}\right|_{R_{n}=i}-V_{i}\left(x_{k_{n}}\right)} x_{k_{n}}^{\prime}\left[\sum_{\tau=1}^{T_{\max }^{i}} \sum_{j \in \mathcal{I}} \pi_{i j}(\tau) A_{i}^{\prime \tau} P_{j} A_{i}^{\tau} / \eta_{i}-P_{i}\right] x_{k_{n}} \\
\leq-\lambda_{\min }\left(-\sum_{\tau=1}^{T_{\max }^{i}} A_{i}^{\prime \tau} \mathcal{P}_{i}(\tau) A_{i}^{\tau}+P_{i}\right)\left\|x_{k_{n}}\right\|^{2} \\
\leq-\beta\left\|x_{k_{n}}\right\|^{2}
\end{gathered}
$$

where $\eta_{i}$ is defined in (11) and $\beta \triangleq \inf _{i \in \mathcal{I}}\left\{\lambda_{\min }\left(-\sum_{\tau=1}^{T_{\max }^{i}}\right.\right.$ $\left.\left.A_{i}^{\prime \tau} \mathcal{P}_{i}(\tau) A_{i}^{\tau}+P_{i}\right)\right\}$. Then, by (12), (13), (14) and Lemma 1 , it follows that S-MJLS (1) is mean-square stable provided that the upper bound of sojourn-time is $T_{\max }^{i}$, i.e., (4) holds, thus the $\sigma$-MSS of the system is guaranteed.

Note that the Lyapunov function of each mode is not necessarily monotonically decreasing $\left(h_{i}\right.$ can be greater than 1$)$, as shown in (10). An illustration of Theorem 1 for $M=3$ is given in Fig. 2, where the system at $k_{n}$ is supposed to run in mode 1 . In $\left(\tau, V_{i}\right)$-coordinate, $V_{2}\left(x_{k_{n}+\tau}\right)$ and/or $V_{3}\left(x_{k_{n}+\tau}\right)$ (the corresponding hollow square or triangle) can be greater than $V_{1}\left(x_{k_{n}}\right)$ (the solid circle in $\left(k, V_{i}\right)$ coordinate). However, as long as the expectation $\bar{V}_{i}\left(x_{k_{n+1}}\right)$ is lower than $V_{i}\left(x_{k_{n}}\right)$, the value of Lyapunov function at jumping instants will tend to zero in stochastic sense, forcing $V_{i}\left(x_{k}\right) \rightarrow 0$, despite the allowable increase of Lyapunov function associated with mode 1 to some extent.

Remark 4: Combining with Remark 3, one can conclude from Theorem 1 that the unforced S-MJLSs will be mean-square stable when $T_{\max }^{i} \rightarrow \infty, \forall i \in \mathcal{I}$, but obviously the resulting conditions (10), (11) can not be numerically tested. Therefore, a finite $T_{\max }^{i}$ is necessitated in Theorem 1, which corresponds to the proposed $\sigma$-MSS with $\sigma>0$.

Remark 5: In Theorem 1, the $\operatorname{SMK} \Pi(\tau)$ is directly utilized to establish the stability criterion. In fact, $\Pi(\tau)$ can be first used to obtain the memory TPs (if denoted by $\lambda_{i j}(\tau)$ ), and the following stability criterion can be arrived at:

$$
\sum_{j \in \mathcal{I}} \lambda_{i j}(\tau) A_{i}^{\prime} P_{j}(\tau) A_{i}-P_{i}(\tau-1) \prec 0
$$

where $P_{i}(\tau)$ is a time-varying Lyapunov matrix and $\tau \in \mathbb{Z}_{>1}$. Note that when $\lambda_{i j}(\tau) \equiv \lambda_{i j}$ (the corresponding PDF of sojourn-time is 
subject to geometric distribution with parameter $\mu_{i}$, i.e., $\omega_{i 1}(\tau)=$ $\left.\cdots=\omega_{i M}(\tau)=\mu_{i}\left(1-\mu_{i}\right)^{\tau-1} \triangleq \omega_{i}(\tau)\right)$ and $P_{i}(\tau) \equiv P_{i}$, (15) reduces to the usual stability criterion for MJLSs. It can be readily proved (cf. [4]) that (15) is a necessary and sufficient condition for the MSS of the unforced system (1). However, (15) is not numerically testable even conservatively setting $P_{i}(\tau) \equiv P_{i}$. Additional approximation techniques such as taking bounds for $\lambda_{i j}(\tau)$ are needed to make (15) be time-invariant, cf. [9]. Besides, (15) aims at MSS that is not "scaled" compared with $\sigma$-MSS.

Remark 6: It is also worth mentioning that if the sojourn-time is subject to "exponentially modulated periodic (EMP)" distribution (cf. [17]), say $\omega_{i}(\tau)=\bar{\omega}_{i}(\tau)\left(1-\mu_{i}\right)^{\tau-1}$, where $\bar{\omega}_{i}(\tau)$ satisfies $\bar{\omega}_{i}(\tau)=$ $\bar{\omega}_{i}(\tau+T)$ with $T$ being the period, then the TPs obtained from SMK will be periodically time-varying. The corresponding criterion in (15) will be periodic and testable accordingly, i.e., only a finite set of $P_{i}(v)$, $v \in \mathbb{Z}_{[1, T]}$ is required. The benefit in this scenario therefore suggests that the EMP distribution can be considered to approximate to those general "exponential-like" distributions of sojourn-time, for the sake of a finite number of conditions.

Though the stability criterion explored in Theorem 1 can be used for analysis of the $\sigma$-MSS of S-MJLSs, the requirements (10), (11) impose a significant difficulty in deriving tractable conditions of controller design due to the existence of the power of $A_{i}$. To circumvent the difficulty, certain techniques will be further explored below to obtain sufficient conditions for Theorem 1.

Theorem 2: Consider the S-MJLS (1) with $u(k) \equiv 0$ and a given finite constant $h_{i}>0$. If, $\forall i \in \mathcal{I}$, there exist $T_{\max }^{i} \in \mathbb{Z}_{\geq 1}$ and a set of matrices $O_{i}(t, m), \forall t \in \mathbb{Z}_{\left[1, T_{\text {max }}^{i}\right]}, \forall m \in \mathbb{Z}_{[0, t]}$ with $O_{i} \triangleq$ $O_{i}(t, t) \succ 0$ and $\mathcal{O}_{i}(\tau, n), \forall \tau \in \mathbb{Z}_{\left[1, T_{\text {max }}^{i}\right]}, \forall n \in \mathbb{Z}_{[0, \tau-1]}$ such that $\forall t \in \mathbb{Z}_{\left[1, T_{\text {max }}^{i}\right]}, \forall m \in \mathbb{Z}_{[0, t-1]}$ and $\forall n \in \mathbb{Z}_{\left[0, T_{\text {max }}^{i}-1\right]}$

$$
\begin{aligned}
& A_{i}^{\prime} O_{i}(t, m+1) A_{i}-O_{i}(t, m) \prec 0 \\
& O_{i}(t, 0)-h_{i} O_{i} \prec 0 \\
& \sum_{\tau=n+1}^{T_{\max }^{i}}\left[A_{i}^{\prime} \mathcal{O}_{i}(\tau, n+1) A_{i}-\mathcal{O}_{i}(\tau, n)\right] \prec 0 \\
& \sum_{\tau=1}^{T_{\max }^{i}} \mathcal{O}_{i}(\tau, 0)-O_{i} \prec 0
\end{aligned}
$$

where $\mathcal{O}_{i}(l, l) \triangleq \sum_{j \in \mathcal{I}} \pi_{i j}(l) O_{j} / \eta_{i}$ with $\eta_{i}$ defined in (11), then the unforced S-MJLS is $\sigma$-error mean-square stable.

Proof: It follows from (16) that $\sum_{m=0}^{t-1} A_{i}^{\prime m}\left(A_{i}^{\prime} O_{i}(t, m+\right.$ 1) $\left.A_{i}-O_{i}(t, m)\right) A_{i}^{m} \prec 0$ and accordingly

$$
A_{i}^{\prime t} O_{i}(t, t) A_{i}^{t}-O_{i}(t, 0) \prec 0 .
$$

By (17), (20), and bearing in mind $O_{i}=O_{i}(t, t)$, it can be obtained that $A_{i}^{\prime t} O_{i} A_{i}^{t} \prec h_{i} O_{i}$. Further, setting the positive definite matrix $O_{i}=P_{i}$, we have $A_{i}^{\prime t} P_{i} A_{i}^{t}-h_{i} P_{i} \prec 0$.

Likewise, (18) ensures that $\sum_{n=0}^{T_{\max }^{i}-1} A_{i}^{\prime n}\left[\sum_{\tau=n+1}^{T_{\max }^{i}}\left[A_{i}^{\prime} \mathcal{O}_{i}(\tau, n+\right.\right.$ 1) $\left.\left.A_{i}-\mathcal{O}_{i}(\tau, n)\right]\right] A_{i}^{n} \prec 0$, which is equivalent to $\sum_{\tau=1}^{T_{\max }^{i}} \sum_{n=0}^{\tau-1}$ $A_{i}^{\prime n}\left[A_{i}^{\prime} \mathcal{O}_{i}(\tau, n+1) A_{i}-\mathcal{O}_{i}(\tau, n)\right] A_{i}^{n} \prec 0$ and implies

$$
\sum_{\tau=1}^{T_{\max }^{i}}\left[A_{i}^{\prime \tau} \mathcal{O}_{i}(\tau, \tau) A_{i}^{\tau}-\mathcal{O}_{i}(\tau, 0)\right] \prec 0 .
$$

Combining (19) and (21), we have $\sum_{\tau=1}^{T_{\max }^{i}} A_{i}^{\prime \tau} \mathcal{O}_{i}(\tau, \tau) A_{i}^{\tau}-P_{i} \prec$ 0 . Then setting the positive definite matrix $\mathcal{O}_{i}(\tau, \tau)=\mathcal{P}_{i}(\tau)$ gives rise to $\sum_{\tau=1}^{T_{\max }^{i}} A_{i}^{\prime \tau} \mathcal{P}_{i}(\tau) A_{i}^{\tau}-P_{i} \prec 0$. Thus, by Theorem 1 , it can be concluded that the system is $\sigma$-error mean-square stable and this completes the proof.

Then, based on Theorem 2, the existence conditions of modedependent stabilizing controller (6) for S-MJLS (1) are presented in the following theorem.
Theorem 3: Consider the S-MJLS (1) with a given finite constant $h_{i}>0$. If, $\forall i \in \mathcal{I}$, there exist $T_{\max }^{i} \in \mathbb{Z}_{\geq 1}$ and a set of matrices $H_{i}(t, m), \forall t \in \mathbb{Z}_{\left[1, T_{\max }^{i}\right]}, \forall m \in \mathbb{Z}_{[0, t]}$ with $H_{i} \triangleq H_{i}(t, t) \succ 0$ and $\left.\mathcal{H}_{i}(n), \forall n \in \mathbb{Z}_{\left[0, T_{\text {max }}^{i}\right]}\right], Z_{i}, U_{i}$ such that $\forall t \in \mathbb{Z}_{\left[1, T_{\text {max }}^{i}\right]}, \forall m \in$ $\mathbb{Z}_{[0, t-1]}$ and $\forall n \in \mathbb{Z}_{\left[0, T_{\max }^{i}-1\right]}$

$$
\begin{aligned}
& {\left[\begin{array}{cc}
H_{i}(t, m+1)-Z_{i}-Z_{i}^{\prime} & A_{i} Z_{i}+B_{i} U_{i} \\
* & -H_{i}(t, m)
\end{array}\right] \prec 0} \\
& H_{i}(t, 0)-h_{i} H_{i} \prec 0 \\
& {\left[\begin{array}{ccc}
\mathcal{H}-\mathcal{Z}-\mathcal{Z}^{\prime} & 0 & \left(\mathcal{A}_{i} \mathcal{Z}_{i}+\mathcal{B}_{i} \mathcal{U}_{i}\right) \mathcal{L}_{i}(n+1) \\
* & \mathcal{H}_{i}(n+1)-Z_{i}-Z_{i}^{\prime} & \left(A_{i} Z_{i}+B_{i} U_{i}\right) L_{i}(n+1) \\
* & * & -\mathcal{H}_{i}(n)
\end{array}\right] \prec 0} \\
& \mathcal{H}_{i}(0)-H_{i} \prec 0
\end{aligned}
$$

where $\mathcal{A}_{i} \triangleq \operatorname{diag}_{(M)}\left\{A_{i}\right\}, \mathcal{B}_{i} \triangleq \operatorname{diag}_{(M)}\left\{B_{i}\right\}, \mathcal{Z}_{i} \triangleq \operatorname{diag}_{(M)}\left\{Z_{i}\right\}$, $\mathcal{U}_{i} \triangleq \operatorname{diag}_{(M)}\left\{U_{i}\right\}, \quad \mathcal{H} \triangleq \operatorname{diag}\left\{H_{1}, H_{2}, \ldots, H_{M}\right\}, \quad \mathcal{Z} \triangleq$ $\operatorname{diag}\left\{Z_{1}, Z_{2}, \ldots, Z_{M}\right\}, \quad L_{i}(n)=I, \quad \forall n \in \mathbb{Z}_{\left[1, T_{\max }^{i}-1\right]} \quad$ with $L_{i}\left(T_{\max }^{i}\right)=0, \quad$ and $\quad \mathcal{L}_{i}(n) \triangleq\left[\ell_{i 1}(n) I, \ell_{i 2}(n) I, \ldots, \ell_{i M}(n) I\right]^{\prime}$ with $\ell_{i j}(n) \triangleq \sqrt{\pi_{i j}(n) / \eta_{i}}$ and $\eta_{i}$ defined in (11), then a modedependent controller of form (6) can be obtained to guarantee the $\sigma$-MSS of the resulting closed-loop system. Moreover, the admissible controller gain is given by $K_{i}=U_{i} Z_{i}^{-1}$.

Proof: Letting $\overline{\mathcal{O}}_{i}(l) \triangleq \sum_{\tau=l+1}^{T_{\max }^{i}} \mathcal{O}_{i}(\tau, l), \forall l \in \mathbb{Z}_{\left[0, T_{\max }^{i}-1\right]}$ and $\overline{\mathcal{O}}_{i}\left(T_{\text {max }}^{i}\right) \triangleq 0$, we can rewrite (18) and (19) as

$$
\begin{aligned}
\overline{\mathcal{O}}_{i}(0)-O_{i} & \prec 0 \\
A_{i}^{\prime} \mathcal{O}_{i}(n+1, n+1) A_{i}+A_{i}^{\prime} \overline{\mathcal{O}}_{i}(n+1) A_{i}-\overline{\mathcal{O}}_{i}(n) & \prec 0 .
\end{aligned}
$$

Consider (27), by Schur complement, it yields that

$$
\left[\begin{array}{ccc}
-\mathcal{O} & 0 & \mathcal{O} \mathcal{A}_{i} \mathcal{L}_{i}(n+1) \\
* & -\overline{\mathcal{O}}_{i}(n+1) & \overline{\mathcal{O}}_{i}(n+1) A_{i} \\
* & * & -\overline{\mathcal{O}}_{i}(n)
\end{array}\right] \prec 0
$$

where $\mathcal{A}_{i}, \mathcal{L}_{i}(n)$ are defined in (24) and $\mathcal{O} \triangleq \operatorname{diag}\left\{O_{1}, O_{2}, \ldots\right.$, $\left.O_{M}\right\}$. Performing a congruence transformation to (28) by $\operatorname{diag}\left\{\mathcal{O}^{-1} \mathcal{V}\right.$, $\left.\overline{\mathcal{O}}_{i}(n+1)^{-1} V_{i}, I\right\}$, where $\mathcal{V} \triangleq \operatorname{diag}\left\{V_{1}, V_{2}, \ldots, V_{M}\right\}$, and since $(\mathcal{O}-\mathcal{V})^{\prime} \mathcal{O}^{-1}(\mathcal{O}-\mathcal{V}) \succ 0$ ensures $\mathcal{O}-\mathcal{V}-\mathcal{V}^{\prime} \succ-\mathcal{V}^{\prime} \mathcal{O}^{-1} \mathcal{V}$, we can obtain

$$
\left[\begin{array}{ccc}
\mathcal{O}-\mathcal{V}-\mathcal{V}^{\prime} & 0 & \mathcal{V}^{\prime} \mathcal{A}_{i} \mathcal{L}_{i}(n+1) \\
* & \overline{\mathcal{O}}_{i}(n+1)-V_{i}-V_{i}^{\prime} & V_{i}^{\prime} A_{i} \\
* & * & -\overline{\mathcal{O}}_{i}(n)
\end{array}\right] \prec 0 .
$$

Then, apply the congruence transformation to (24) by $\operatorname{diag}\left\{\mathcal{V}, V_{i}, V_{i}\right\}$, it gives (29) while replacing $A_{i}$ by $A_{i}+B_{i} K_{i}$ and setting $Z_{i} \triangleq V_{i}^{-1}$, $U_{i} \triangleq K_{i} V_{i}^{-1}, \mathcal{H}=\mathcal{V}^{\prime-1} \mathcal{O} \mathcal{V}^{-1}, \mathcal{H}_{i}(n) \triangleq V_{i}^{\prime-1} \overline{\mathcal{O}}_{i}(n) V_{i}^{-1}$.

On the other hand, performing a congruence transformation to (25) by $V_{i}$, we have (26) accordingly. Then, it implies that (24) and (25) guarantee (18) and (19) in Theorem 2. Same techniques can be applied to the case that (22) and (23) ensures (16) and (17). Thus, it can be concluded from Theorem 2 that (22)-(25) can guarantee the $\sigma$-MSS of the resulting closed-loop system with the admissible controller gain given by $K_{i}=U_{i} Z_{i}^{-1}$.

Remark 7: In Theorems 1 and 3, for a given set of $h_{i}$, the $T_{\max }^{i}$ can be maximized while achieving a minimal $\sigma$-error and ensuring a feasible solution of (10), (11), and (22)-(25), for stability and stabilization problems, respectively. Besides, note also that if $h_{i}>1$, the corresponding Lyapunov function in the $i$ th mode can raise to some extent, which implies that mode $i$ can be unstable/unstabilizable with $\tau \leq T_{\max }^{i}$. Nevertheless, it can be conjectured that if such an unstable/unstabilizable mode is expected to run longer (i.e., a larger $\left.T_{\max }^{i}\right)$, then probably a much larger $h_{i}\left(h_{i} \gg 1\right)$ is required, which will result in a huge energy growth. 
TABLE I

The Optimal $T_{\max }^{i}$ And the Minimal $\sigma$-ERror For Given Different $h_{i}, i=1,2$

\begin{tabular}{|c|c|c|c|c|c|c|c|c|c|}
\hline & \multicolumn{2}{|c|}{$\alpha=1.07$} & \multicolumn{2}{|c|}{$\alpha=1.15$} & \multicolumn{2}{|c|}{$\alpha=1.20$} & \multicolumn{2}{|c|}{$\alpha=1.27$} & $\alpha=1.28$ \\
\hline$h_{i}$ & $T_{\max }^{1}, T_{\max }^{2}$ & $\sigma$ & $T_{\max }^{1}, T_{\max }^{2}$ & $\sigma$ & $T_{\max }^{1}, T_{\max }^{2}$ & $\sigma$ & $T_{\max }^{1}, T_{\max }^{2}$ & $\sigma$ & \\
\hline 3 & 3,6 & 0.26 & 3,4 & 0.31 & 2,2 & 0.73 & 1,1 & 1.61 & \\
\hline 5 & 5,7 & 0.09 & 4,3 & 0.27 & 2,3 & 0.58 & 1,1 & 1.61 & infeasible \\
\hline 10 & 6,10 & 0.05 & 4,5 & 0.17 & 2,5 & 0.48 & 1,1 & 1.61 & inteasible \\
\hline $10^{2}$ & 12,20 & $2.2 \times 10^{-3}$ & 4,8 & 0.14 & 2,5 & 0.48 & 1,1 & 1.61 & \\
\hline $10^{3}$ & 18,30 & $0.1 \times 10^{-3}$ & 4,8 & 0.14 & 2,5 & 0.48 & 1,1 & 1.61 & \\
\hline
\end{tabular}

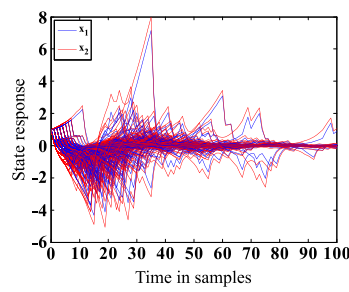

(a)

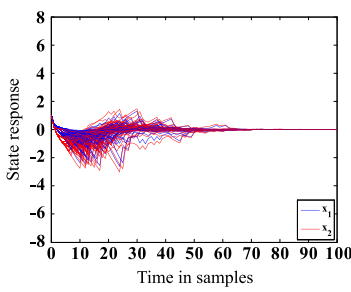

(b)

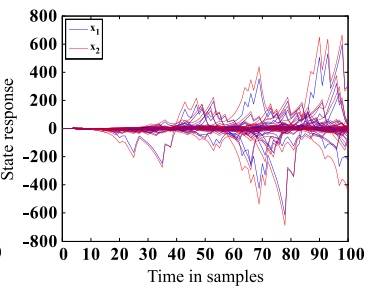

(c)

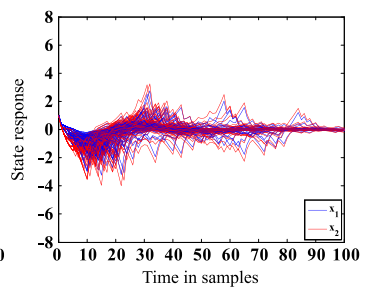

(d)

Fig. 3. 100 realizations of state response of the system when generating different random jumping sequences. In (b) and (d), the sequences are further subject to $T_{\max }^{1}=5, T_{\max }^{2}=7$ and $T_{\max }^{1}=4, T_{\max }^{2}=3$, respectively. (a) $\alpha=1.07$ (not MSS). (b) $\alpha=1.07$ ( $\sigma$-MSS with $\sigma$-error $=0.09$ ). (c) $\alpha=1.15$ (not MSS). (d) $\alpha=1.15$ ( $\sigma$-MSS with $\sigma$-error $=0.27)$.

\section{NumERICAL EXAMPLES}

In this section, both a MJLS and a S-MJLS will be provided to show the validity and advantage of the obtained theoretical results.

Example 1: (MJLS) Consider an unforced MJLS with two modes

$$
A_{1}=\alpha\left[\begin{array}{ll}
-0.36 & 0.69 \\
-1.81 & 1.97
\end{array}\right] \quad A_{2}=\alpha\left[\begin{array}{cc}
0.34 & 0.62 \\
-0.37 & 1.36
\end{array}\right]
$$

where $\alpha>0$ characterizes the distance of system eigenvalues and unit circle. The EMC is an anti-identity matrix, and the sojourn-time is subject to geometric distribution with $\omega_{1}(\tau)=0.4(1-0.4)^{\tau-1}$ and $\omega_{2}(\tau)=0.5(1-0.5)^{\tau-1}$.

Based on the usual criterion of MSS (the required TPs matrix can be straightforwardly obtained from $\omega_{1}(\tau)$ and $\omega_{2}(\tau)$ ), it can be readily checked that the system is not mean-square stable when $\alpha \geq 1.07$. Turning to Theorem 1, given different $h_{i}$, we can maximize the upper bound of sojourn-time for each mode (denoted as $\left[T_{\max }^{i}\right]_{o p t}, i=1,2$ ) in achieving a minimal $\sigma$-error of approximation to MSS, as computed in Table I. It can be seen that when $\alpha>1.27$, no matter how large the set of $h_{i}$ is given, a finite $\sigma$-error for the MSS cannot be guaranteed (the corresponding system does not hold $\sigma$-MSS). As for $1.07 \leq \alpha \leq 1.27$, however, different minimal $\sigma$-error can be found for different $h_{i}$. Particularly, the smaller the $\alpha$ is assigned, the larger the $\left[T_{\max }^{i}\right]_{o p t}$ can be found, and thus the smaller the minimal $\sigma$-error can be achieved.

Consider $\alpha=1.07, x_{0}=\left[\begin{array}{ll}1 & 1\end{array}\right]^{\prime}$, Fig. 3(a) illustrates the state response when randomly generating 100 realizations of jumping sequences satisfying the geometric distribution, and Fig. 3(b) presents the case where the jumping sequences are further subject to $T_{\max }^{1}=$ $5, T_{\max }^{2}=7$. The scenario of $\alpha=1.15$ is also given in Fig. 3(c) and (d) $\left(T_{\max }^{1}=4, T_{\max }^{2}=3\right)$. It can be observed that although the two systems are not mean-square stable, they are $\sigma$-error meansquare stable with different approximation $\sigma$-error to be 0.09 and 0.27 , respectively. Both Table I and Fig. 3 therefore demonstrate that the proposed $\sigma$-MSS is of the advantage of being well-scaled rather than MSS.

Example 2: (S-MJLS) Consider a dynamic system with possible failures in both structure and actuator as

$$
\left\{\begin{array}{l}
A_{1}=\left[\begin{array}{ll}
-0.36 & 0.69 \\
-1.81 & 1.97
\end{array}\right] B_{1}=\left[\begin{array}{r}
-0.1 \\
0.1
\end{array}\right] \\
A_{2}=\left[\begin{array}{rr}
0.34 & 0.62 \\
-0.37 & 1.36
\end{array}\right] B_{2}=\left[\begin{array}{l}
0.1 \\
0.1
\end{array}\right] \\
A_{3}=\left[\begin{array}{rr}
0.34 & 0.62 \\
-0.37 & 1.36
\end{array}\right] B_{3}=\left[\begin{array}{l}
0 \\
0
\end{array}\right]
\end{array}\right.
$$

(Normal Case)

(Slight Fault)

(Serious Fault (unworkable actuator))
The switching among the three modes is governed by a SMC, where

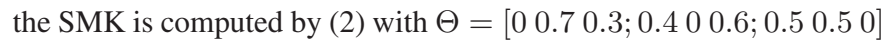
and, $\forall i, j \in\{1,2,3\}$

$$
\left[\omega_{i j}(\tau)\right]=\left[\begin{array}{ccc}
0 & \frac{0.6^{\tau} \cdot 0.4^{10-\tau} \cdot 10 !}{(10-\tau) ! \tau !} & \frac{0.4^{\tau} \cdot 0.6^{10-\tau} \cdot 10 !}{(10-\tau) ! \tau !} \\
0.9^{(\tau-1)^{2}}-0.9^{\tau^{2}} & 0 & \frac{0.5^{10} \cdot 10 !}{(10-\tau) ! \tau !} \\
0.4^{(\tau-1)^{1.3}}-0.4^{\tau^{1.3}} & 0.3^{(\tau-1)^{0.8}}-0.3^{\tau^{0.8}} & 0
\end{array}\right] .
$$

Note from $\omega_{i j}(\tau)$ that the "hybrid" PDF of sojourn-time in mode 1 and mode 3 are considered as Bernoulli distribution and Weibull distribution, respectively, with different parameters when the target modes are different, and the two types of distributions are supposed to coexist in mode 2 .

First, it is checked that the open-loop system is not $\sigma$-error meansquare stable for any $h_{i}>0$ by Theorem 1. Applying Theorem 3, the desired mode-dependent controllers can be designed such that the resulting closed-loop systems are $\sigma$-error mean-square stable for certain sets of $h_{i}$. Also, [ $\left.T_{\max }^{i}\right]_{o p t}$ can be further obtained to achieve a minimal $\sigma$-error for the MSS of the system and ensuring a feasible solution of the controller. It can be verified that no matter how large $h_{1}$ and $h_{2}$ are assigned, no feasible controllers can be obtained if $h_{3}<1$. Thus, consider $h_{3} \geq 1$ and change $h_{1}, h_{2}$, the different $\left[T_{\max }^{i}\right]_{\text {opt }}$ can be obtained. Fig. 4(a)-(c) present the two sets of $\left[T_{\max }^{i}\right]_{\text {opt }}$ that vary with $h_{2} \in \mathbb{R}_{[0.001,0.005]}$ and $h_{3} \in \mathbb{R}_{[1.1,1.5]}$, for given $h_{1}$ to be 0.01 and 0.001 , respectively [the corresponding $\sigma$-error, which can be computed by (5), is shown in Fig. 4(d)].

Then, setting $h_{1}=0.01, h_{2}=0.005$ and $h_{3}=1.5$, the upper bound of sojourn-time can be optimized as $\left[T_{\max }^{1}\right]_{o p t}=\left[T_{\max }^{2}\right]_{o p t}=$ 9 and $\left[T_{\max }^{3}\right]_{o p t}=3$, which guarantee the minimal $\sigma$-error to be 0.0467 . Given the initial condition $x_{0}=[-0.51]^{\prime}$, Fig. 5 shows the 100 realizations of the state response of the closed-loop system for randomly generating jumping sequences satisfying (the required $T_{\max }^{i}$ ) $T_{\max }^{1}=T_{\max }^{2}=9$ and $T_{\max }^{3}=3$. It can be observed that the designed controller is valid in the presence of an unstabilizable mode and despite the coexistence of "hybrid" distributions of sojourn-time in a same SMK. In addition, as shown in Fig. 4(d), when fixing $T_{\max }^{1}=T_{\max }^{2}=9$, the $\sigma$-error can be smaller if further increasing $T_{\max }^{3}$, which seemingly can be attained by raising $h_{i}$. However, it can be checked that even $h_{i}=10^{7}$ can not give rise to an increase from $T_{\max }^{3}=3$ to $T_{\max }^{3}=4$, which verifies the conjecture in Remark 7 that only a proper sojourn-time of the unstabilizable mode can be allowed. 


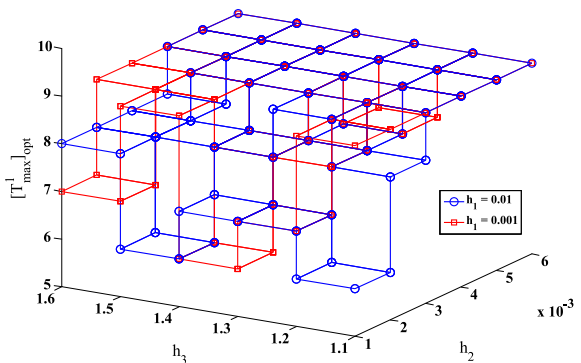

(a)

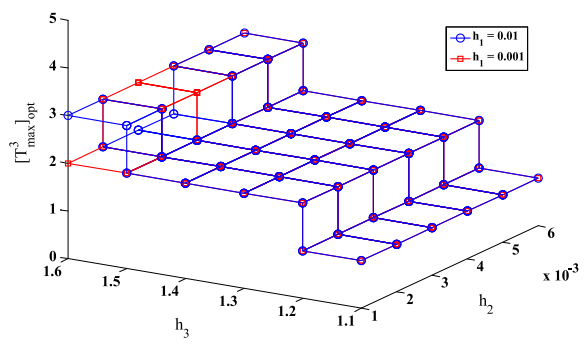

(c)

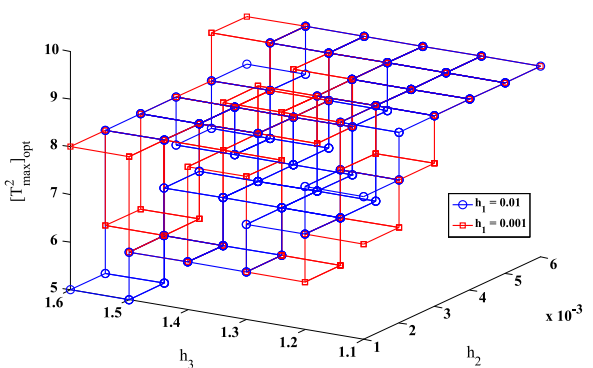

(b)

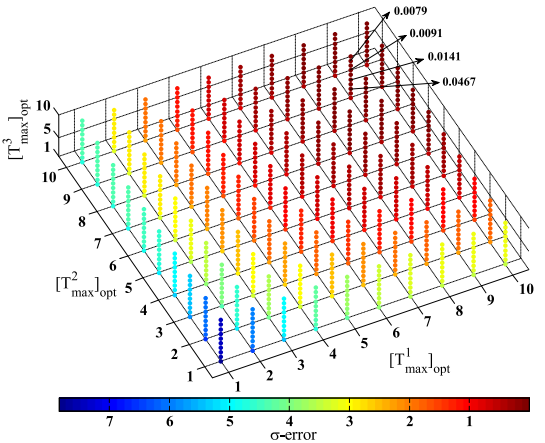

(d)

Fig. 4. Optimal $T_{\max }^{i}$ varying with $h_{i}$ and the $\sigma$-error varying with $T_{\max }^{i}, i=1,2,3$ (note that in (a)-(c), four squares or circles in the horizontal specify a face where the $T_{\max }^{i}$ is constant, e.g., in (a), for $0.001 \leq h_{2} \leq 0.002$, and $1.5 \leq h_{3} \leq 1.6, T_{\max }^{1}$ is 8 and 7 for $h_{1}=0.01$ and $h_{1}=0.001$, respectively). (a) Variation of optimal $T_{\max }^{1}$. (b) Variation of optimal $T_{\max }^{2}$. (c) Variation of optimal $T_{\max }^{3}$. (d) $\sigma$-error varying with $T_{\max }^{i}$.

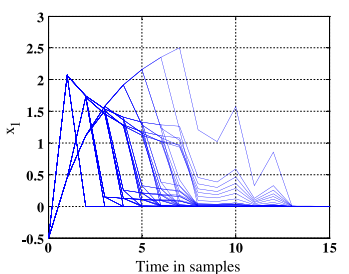

(a)

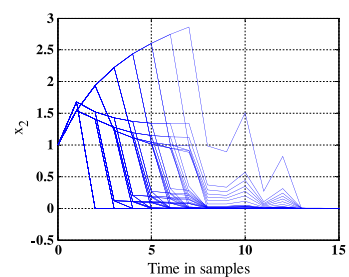

(b)
Fig. 5. 100 realizations of state response for different random jumping sequences satisfying $T_{\max }^{1}=T_{\max }^{2}=9$ and $T_{\max }^{3}=3$. (a) State response $x_{1}$. (b) State response $x_{2}$.

\section{CONCLUSION}

In this technical note, the stability and stabilization problems of discrete-time S-MJLSs were investigated. The notion of discrete-time SMK is introduced, and the PDF of sojourn-time inside the SMK can be considered to be dependent on both current and next system mode. The underlying S-MJLSs are therefore more general than existing ones, since different parameters in a same type of distribution and/or different types of distributions can coexist in each mode of a SMK. The stability concept of $\sigma$-MSS was proposed, where $\sigma$ is capable of characterizing the degree of approximation error of $\sigma$-MSS to MSS. Numerically testable stability and stabilization criteria that explicitly contain the PDF information of sojourn-time were obtained. A direct future work will be the developments of the S-MJLSs in continuoustime domain based on the SMK approach.

\section{REFERENCES}

[1] M. Mahmoud, J. Jiang, and Y. Zhang, "Stochastic stability analysis of fault-tolerant control systems in the presence of noise," IEEE Trans. Autom. Control, vol. 46, no. 11, pp. 1810-1815, Nov. 2001.

[2] L. Zhang, Y. Shi, T. Chen, and B. Huang, "A new method for stabilization of networked control systems with random delays," IEEE Trans. Autom. Control, vol. 50, no. 8, pp. 1177-1181, Aug. 2005.
[3] X. Feng, K. A. Loparo, Y. Ji, and H. J. Chizeck, "Stochastic stability properties of jump linear systems," IEEE Trans. Autom. Control, vol. 37, no. 1, pp. 38-53, Jan. 1992.

[4] Y. Ji and H. J. Chizeck, "Jump linear quadratic gaussian control: Steadystate solution and testable conditions," Control-Theory Adv. Technol., vol. 6, no. 3, pp. 289-319, 1990.

[5] P. Shi, E.-K. Boukas, and R. K. Agarwal, "Control of Markovian jump discrete-time systems with norm bounded uncertainty and unknown delay," IEEE Trans. Autom. Control, vol. 44, no. 11, pp. 2139-2144, Nov. 1999.

[6] P. Bolzern, P. Colaneri, and G. De Nicolao, "Stochastic stability of positive Markov jump linear systems," Automatica, vol. 50, no. 4, pp. 1181-1187, 2014.

[7] O. L. do Valle Costa, M. D. Fragoso, and R. P. Marques, Discrete-Time Markov Jump Linear Systems. New York, NY, USA: Springer-Verlag, 2006.

[8] C. Schwartz and A. H. Haddad, "Control of jump linear systems having semi-Markov sojourn times," in Proc. 42nd IEEE Conf. Decision Control, 2003, vol. 3, pp. 2804-2805.

[9] J. Huang and Y. Shi, "Stochastic stability and robust stabilization of semiMarkov jump linear systems," Int. J. Robust Nonlin. Control, vol. 23, pp. 2028-2043, 2013.

[10] P. Bolzern, P. Colaneri, and G. De Nicolao, "Markov jump linear systems with switching transition rates: Mean square stability with dwell-time," Automatica, vol. 46, no. 6, pp. 1081-1088, 2010.

[11] L. Zhang, " $H_{\infty}$ estimation for discrete-time piecewise homogeneous Markov jump linear systems," Automatica, vol. 45, no. 11, pp. 2570-2576, 2009.

[12] S. Aberkane, "Stochastic stabilization of a class of nonhomogeneous Markovian jump linear systems," Syst. Control Lett., vol. 60, no. 3, pp. $156-160,2011$.

[13] R. A. Howard, "System analysis of semi-Markov processes," IEEE Trans. Military Electron., vol. ME-8, no. 2, pp. 114-124, 1964.

[14] L. Campo, P. Mookerjee, and Y. Bar-Shalom, "State estimation for systems with sojourn-time-dependent Markov model switching," IEEE Trans. Autom. Control, vol. 36, no. 2, pp. 238-243, 1991.

[15] V. Barbu and N. Limnios, "Empirical estimation for discrete-time semiMarkov processes with applications in reliability," Nonparametric Statist., vol. 18, no. 7-8, pp. 483-498, 2006.

[16] E.-K. Boukas and Z. Liu, "Robust $H_{\infty}$ control of discrete-time Markovian jump linear systems with mode-dependent time-delays," IEEE Trans. Autom. Control, vol. 46, no. 12, pp. 1918-1924, Dec. 2001.

[17] S. Bittanti and P. Colaneri, Periodic Systems: Filtering and Control. New York, NY, USA: Springer-Verlag, 2008. 